\title{
A Review and Analysis of Software Complexity Metrics in Structural Testing
}

\author{
Mrinal Kanti Debbarma, Swapan Debbarma, Nikhil Debbarma, Kunal Chakma, and Anupam Jamatia
}

\begin{abstract}
Software metrics is developed and used by the various software organizations for evaluating and assuring software code quality, operation, and maintenance. Software metrics measure various types of software complexity like size metrics, control flow metrics and data flow metrics. These software complexities must be continuously calculated, followed, and controlled. One of the main objectives of software metrics is that applies to a process and product metrics. It is always considered that high degree of complexity in a module is bad in comparison to a low degree of complexity in a module. Software metrics can be used in different phases of the software development lifecycle. This paper reviews the theory, called "software complexity metrics", and analysis has been done based on static analysis. We try to evaluate and analyze different aspects of software metrics in structural testing which offers of estimating the effort needed for testing.
\end{abstract}

Index Terms-Software metrics, lines of code, control flow metrics, NPATH complexity, structural testing.

\section{INTRODUCTION}

The Software complexity is based on well-known software metrics, this would be likely to reduce the time spent and cost estimation in the testing phase of the software development life cycle (SDLC), which can only be used after program coding is done. Improving quality of software is a quantitative measure of the quality of source code. This can be achieved through definition of metrics, values for which can be calculated by analyzing source code or program is coded. A number of software metrics widely used in the software industry are still not well understood [1]. Although some software complexity measures were proposed over thirty years ago and some others proposed later. Sometimes software growth is usually considered in terms of complexity of source code. Various metrics are used, which unable to compare approaches and results. In addition, it is not possible or equally easy to evaluate for a given source code [2]. Software complexity, deals with how difficult a program is to comprehend and work with [3]. Software maintainability [3], is the degree to which characteristics that hamper software maintenance are present and determined by software complexity. There dependencies are shown in Fig. 1.

This paper presents an analysis by which tester/developer can minimize software development cost and improve testing efficacy and quality

Manuscript received August 16, 2012; revised September 28, 2012.

The authors are with the Department of Computer Science \& Engineering, National Institute of Technology, Agartala, Tripura, INDIA, PIN 799055, India (e-mail: mrinal@nita.ac.in).

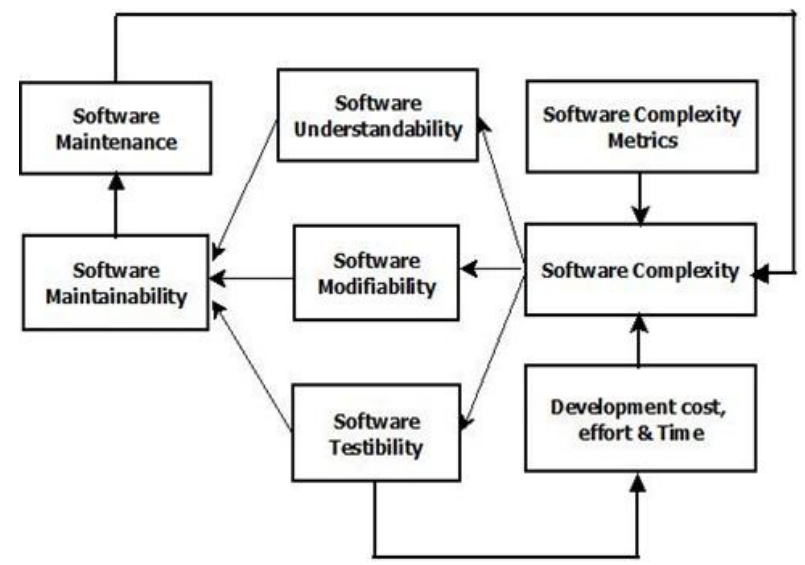

Fig. 1. Relationship between software complexity metrics and software systems

\section{Problem Statement}

From software engineering point of view software development experience shows, that it is difficult to set measurable targets when developing software products. Produced/developed software has to be testable, reliable and maintainable. On the other side, "You cannot control what you cannot measure" [4]. In software engineering field during software process, developers do not know if what they are developing is correct and guidance are needed to help them accustom more improvement. Software metrics are facilitating to track software enhancement. Various industries dedicated to develop software, and use software metrics in a regular basis. Some of them have produced their own standards of software measurement, so the use of software metrics is totally depending upon industry to industry. In this regards, what to measure is classified into two categories, such that software process or software product. But ultimately, main goal of this measure is customer satisfaction not only at delivery, but through the whole development process.

\section{BACKGORUND AND RELATED WORK}

\section{A. Software Metrics}

Software metrics is defined by measuring of some property of a portion of software or its specifications. Software metrics provide quantitative methods for assessing the software quality. Software metrics can be define as: "The continuous application of measurement-based techniques to the software development process and its products to supply meaningful and timely management information (MI) together with the use of those techniques to improve its products and that process" [5]. 


\section{B. Software Complexity}

Software complexity, deals with how difficult a program is to comprehend and work with [3]. Software maintainability [3], is the degree to which characteristics that hamper software maintenance are present and determined by software complexity. Software complexity is based on well-known software metrics.

Various software complexity metrics invented and can be categorized into two types:

\section{1) Static metrics}

Static metrics are obtainable at the early phases of software development life cycle (SDLC).

These metrics deals with the structural feature of the software system and easy to gather.

Static complexity metrics estimate the amount of effort needed to develop, and maintain the code.

\section{2) Dynamic metrics}

Dynamic metrics are accessible at the late stage of the software development life cycle (SDLC). These metrics capture the dynamic behavior of the system and very hard to obtain and obtained from traces of code.

\section{Software Complexity Measures: Attributes}

Software complexity metrics can be distinguished by the attributes used for measurement. In this paper, we are concentrating on static measure which can be classified into three types:

1) Size based metrics

Size is one of the most essential attributes of software systems [6]. It controls the expenditure incurred for the systems both in man-power and budget, for the development and maintenance. These metrics specify the complexity of software by size attributes and helps in predicting the cost involvement for maintaining the system. Size based metrics measures the actual size of the software module. Metrics is originated from the basic counts such as line numbers, volume, size, effort, length, etc.

\section{2) Control flow based metrics}

Control flow based metrics measures the comprehensibility of control structures. These metrics also confine the relation between the logic structures in program with its program complexity. These metrics are originated from the control structure of a program [3].

\section{3) Data flow based metrics}

Data flow based metrics measure the usage of data and their data dependency (visibility of data as well as their interactions) [3].

Structural testing criteria consider on the knowledge of the internal structure of the program implementation to derive the testing criteria. Test cases are generated for actual implementation, if there is some change in implementation then it leads to change in test cases. They can be classified as, complexity, control flow and data flow based criteria. The complexity based criterion requires the execution of all independent paths of the program; it is based on McCabe's complexity concept [7]. For the control flow based criteria, testing requirements are based on the Control Flow Graph (CFG). It requires the execution of components (blocks) of the program under test in condition of subsequent elements of the CFG i.e. nodes, edges and paths. Another method is number of unit tests needed to test every combination of paths in a method. In Data Flow based criteria, both data flow and control flow information are used to perform testing requirements. These coverage criteria are based on code coverage. Code coverage is the degree to which source code of a program has been tested. Test coverage is measured during test execution. Once such a criterion has been selected, test data must be selected to fulfill the criterion.

TABLE I: SUMMARIZED SOFTWARE METRICS ARE SHOWN IN.

\begin{tabular}{|c|c|c|c|}
\hline Type & Metrics & Description & $\begin{array}{l}\text { Merit \& } \\
\text { De-merits }\end{array}$ \\
\hline $\begin{array}{l}\text { Size } \\
\text { Metrics } \\
\text { (Program } \\
\text { Size) }\end{array}$ & $\begin{array}{l}\text { Lines of Code } \\
\text { (LOC), } \\
\text { Token Counts } \\
\text { (TC), } \\
\text { Function Points } \\
\text { (FP), } \\
\text { Halstead's } \\
\text { software } \\
\text { science (HSS) }\end{array}$ & $\begin{array}{l}\text { Metrics based on } \\
\text { program size, } \\
\text { amount of lines of } \\
\text { code, } \\
\text { declarations, } \\
\text { statements, and } \\
\text { files. } \\
\text { Halstead's metrics } \\
\text { are based on count } \\
\text { of unique number } \\
\text { of operators and } \\
\text { operands in a } \\
\text { program. }\end{array}$ & $\begin{array}{l}\text { Easy to } \\
\text { understand; } \\
\text { fast to count, } \\
\text { program } \\
\text { language } \\
\text { independent } \\
\text { and widely } \\
\text { applicable. } \\
\text { No need of } \\
\text { deep analysis } \\
\text { of program's } \\
\text { logic } \\
\text { structure. In } \\
\text { contrast } \\
\text { ignores the } \\
\text { complexity } \\
\text { from the } \\
\text { control flow. }\end{array}$ \\
\hline $\begin{array}{l}\text { Control } \\
\text { flow based } \\
\text { metrics } \\
\text { (Program } \\
\text { Control } \\
\text { Structure) }\end{array}$ & $\begin{array}{l}\text { McCabe's } \\
\text { Cyclomatic } \\
\text { Complexity } \\
\text { (MCC), Conte's } \\
\text { Average } \\
\text { Nesting Level, } \\
\text { (CANC), } \\
\text { NPATH } \\
\text { Complexity } \\
\text { (NC) }\end{array}$ & $\begin{array}{l}\text { Metrics based on } \\
\text { control structure } \\
\text { of the program or } \\
\text { control flow graph } \\
\text { (CFG) and density } \\
\text { of control within } \\
\text { the program } \\
\text { Measure acyclic } \\
\text { execution path } \\
\text { through a } \\
\text { program. }\end{array}$ & $\begin{array}{l}\text { Ignores the } \\
\text { complexity } \\
\text { from the data } \\
\text { flow of the } \\
\text { program and } \\
\text { Complexity } \\
\text { added by the } \\
\text { nesting levels. } \\
\text { Do not } \\
\text { distinguish the } \\
\text { complexities } \\
\text { of various } \\
\text { kinds of } \\
\text { control flow. }\end{array}$ \\
\hline $\begin{array}{l}\text { Data Flow } \\
\text { based } \\
\text { metrics }\end{array}$ & $\begin{array}{ll}\text { Chung's live } \\
\text { definition }\end{array}$ & $\begin{array}{l}\text { Metrics is based } \\
\text { on use of data } \\
\text { within a program. }\end{array}$ & $\begin{array}{l}\text { Intra and inter } \\
\text { module's data } \\
\text { dependency } \\
\text { complexity }\end{array}$ \\
\hline
\end{tabular}

Complexity of software is measuring of software code quality; it requires a model to convert internal quality attributes to code reliability. High degree of complexity in a component like function, subroutine, object, class etc. is consider bad in comparison to a low degree of complexity in a component. Software complexity measures which enables the tester to counts the acyclic execution paths through a component and improve software code quality. In a program characteristic that is one of the responsible factors that affect the developer's productivity [8] in program comprehension, maintenance, and testing phase. There are several methods to calculate complexity measures were investigated, e.g., Nesting Level [6], different version of LOC [8], NPATH [9], McCabe's cyclomatic number [10], Data quality [10], Halstead's software science [11], Function Points[12], Token Counts[11], Chung's live definition [13] etc.

\section{Clasification of Software Metrics}

Software metrics are useful to the software process, and product metrics. Various classification of software metrics are as follows: 
1) Software Process metrics

2) Software Product metrics

\section{A. Software Process Metrics}

Software process metrics involves measuring of properties of the development process and also known as management metrics. These metrics include the cost, effort, reuse, methodology, and advancement metrics. Also determine the size, time and number of errors found during testing phase of the SDLC.

\section{B. Software Product Metrics}

Software process metrics involves measuring the properties of the software and also known as quality metrics. These metrics include the reliability, usability, functionality, performance, efficacy, portability, reusability, cost, size, complexity, and style metrics. These metrics measure the complexity of the software design, size or documentation created.

\section{1) Size metrics: Lines of code}

The size of the program indicates the development complexity, which is known as Lines of Code (LOC). The simplest measure of software complexity recommended by Hatton (1977). This metric is very simple to use and measure the number of source instruction required to solve a problem. While counting a number of instructions (source), line used for blank and commenting lines are ignored. The size, complexity of today's software systems demands the application of effective testing techniques. Size attributes are used to describe physical magnitude, bulk etc. Lines of code and Halstead's software science [11] are examples of size metrics. M. Halstead proposed a metrics called software science.

\section{2) Control flow metrics: NPATH complexity [9]}

The control flow complexity metrics are derived from the control structure of a program. The control flow measure by NPATH, invented by Nejmeh [9] it measures the acyclic execution paths, NPATH is a metric which counts the number of execution path through a functions. NPATH is an example of control flow metrics.

One of the popular software complexity measures NPATH complexity (NC) is determined as:

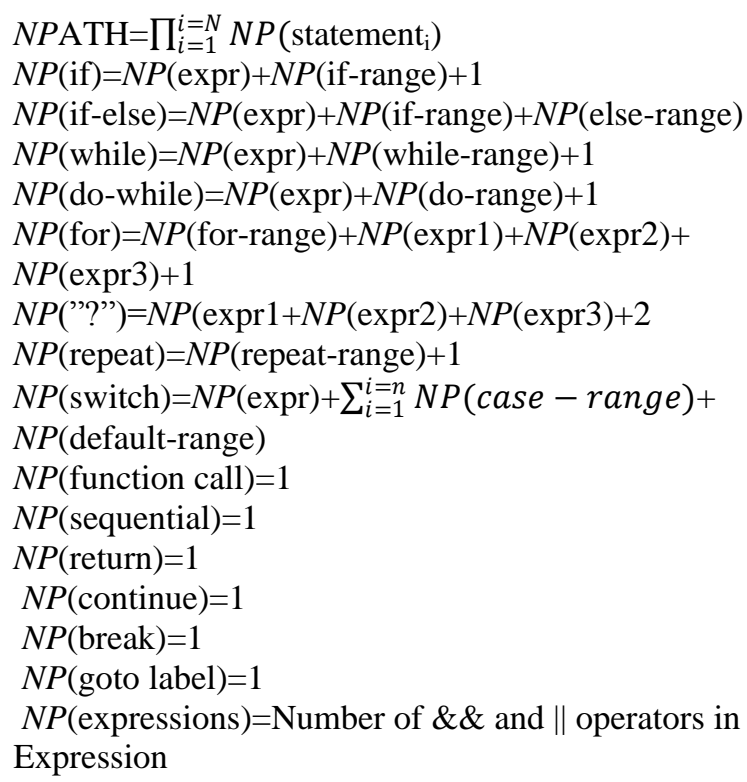

Execution of Path Expressions (complexity expression) are expressed, where " $N$ " represents the number of statements in the body of component (function and "NP (Statement)" represents the acyclic execution path complexity of statement $i$. where "(expr)" represents expression which is derived from flow-graph representation of the statement. For example NPATH measure as follows:

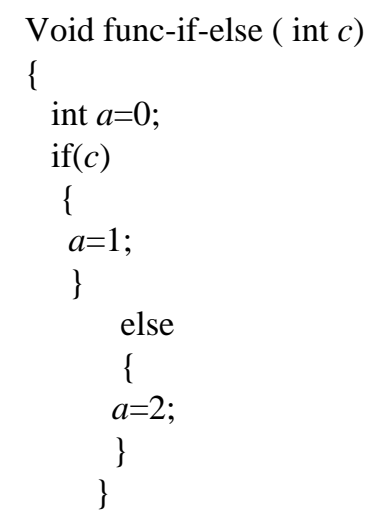

The Value of NPATH $=2$ as follows: $N P($ if-else $)=N P($ expr $)+N P($ if-range $)+N P($ else-range $)$ In the above example, $N P(\exp )=0$ for if statement. $N P$ (If-range) $=1$ for if statement and , $N P($ else-range $)=1$ for if-else statement. So, $N P$ (if-else) $=0+1+1=2$.

NPATH, metric of software complexity overcomes the shortfalls of McCabe's metric which fail to differentiate between various kinds of control flow and nesting levels control structures.

3) Mc cabb'e cyclomatic complexity [10]

Cyclomatic Number is one of the metric based on not program size but more on information/control flow. It is based on specification flow graph representation developed by Thomas J Mc Cabb in 1976. Program graph is used to depict control flow. Nodes are representing processing task (one or more code statement) and edges represent control flow between nodes. McCabe's metrics [7] is example of control flow metrics. To compute Cyclomatic Number by $V$ $(G)$ as following methods:

$$
V(G)=E-N+2 P
$$

where, $V(G)=$ Cyclomatic Complexity

$E=$ the number of edges in a graph

$N=$ the number of nodes in graph

$P=$ the number of connected components in graph,

We can compute the number of binary node (predicate), by the following equation.

$$
V(G)=p+1
$$

where, $V(G)=$ Cyclomatic Complexity $P=$ number of nodes or predicates

The problem with McCabb's Complexity is that, it fails to distinguish between different conditional statements (control flow structures). Also does not consider nesting level of various control flow structures. NPATH, have advantages over the McCabb's metric [12].

4) Halstead software science complexity

M. Halstead's [11] introduced software science measures 
for software complexity product metrics. Halseatd's software science is based on a enhancement of measuring program size by counting lines of code. Halstead's metrics measure the number of number of operators and the number of operands and their respective occurrence in the program (code). These operators and operands are to be considered during calculation of Program Length, Vocabulary, Volume, Potential Volume, Estimated Program Length, Difficulty, and Effort and time by using following formulae.

$n_{1}=$ number of unique operators,

$n_{2}=$ number of unique operands,

$N_{1}=$ total number of operators, and

$N_{2}=$ total number of operands,

a) Program Length $(N)=N 1+N 2$

b) Program Vocabulary $(n)=n 1+n 2$

c) Volume of a Program $(V)=N^{*} \log _{2} \mathrm{n}$

d) Potential Volume of a Program $\left(V^{*}\right)=\left(2+n_{2}\right) \log _{2}\left(2+n_{2}\right)$

e) Program Level $(L)=L=V^{*} / V$

f) Program Difficulty $(D)=1 / L$

g) Estimated Program Length $(N)=n_{1} \log _{2} n_{1}+n_{2} \log _{2} n_{2}$

h) Estimated Program Level $(L)=2 n_{2} /\left(n_{1} N 2\right)$

i) Estimated Difficulty $(D)=1 / L=n_{1} N_{2} / 2 n_{2}$

j) Effort $(E)=V / L=V^{*} D=\left(n_{1} \times N_{2}\right) / 2 n_{2}$

k) Time $(T)=E / S$ [ " $S$ " is Stroud number (given by John

Stroud), the constant " $S$ " represents the speed of a

programmer. The value " $S$ " is 18]

One major weakness of this complexity is that they do not measure control flow complexity and difficult to compute during fast and easy computation.

\section{Static AnAnysis}

Our analysis is based on static analysis of software complexity metrics like size and control flow metrics. We have considered four program characteristics from the literature that are responsible for complexity measures. e.g., LOC, NC, MCC, and HSSC. For this study, we have selected only program written in $\mathrm{C}$ language given in Fig. 2. We have measured LOC, NPATH i.e. acyclic execution paths through components for in an attempt at program optimization, McCabe complexity and finally Halstead's software science complexity metrics. Statics analysis of metrics is not directly associated to the execution of programs (source code). There are three aspects can be affect maintenance of program, like program volume/size, data organization, and control structure.

While counting a number of instructions (source), line used for blank and commenting lines are ignored. NPATH measures the acyclic execution paths which counts the number of execution path through a functions. Halstead's metrics measure the number of number of operators and the number of operands and their respective occurrence in the program (code). These operators and operands are to be considered during calculation of Program Length, Vocabulary, Volume, Potential Volume, Estimated Program Length, Difficulty, and Effort and time. For McCabb's complexity measures program graph is used to depict control flow. Nodes are representing processing task (one or more code statement) and edges represent control flow between nodes

TABLE II: CALCULATION OF THE COMPLEXITY MEASURES FROM PROGRAM $\mathrm{P}$

\begin{tabular}{|c|c|c|c|c|c|}
\hline LOC & NC & MCC & & HSSC & \\
\hline \multirow{9}{*}{35} & \multirow{9}{*}{24} & \multirow[b]{2}{*}{ Nodes } & \multirow[b]{2}{*}{15} & $n 1$ & 15 \\
\hline & & & & $n 2$ & 8 \\
\hline & & \multirow[b]{2}{*}{ Edges } & \multirow[b]{2}{*}{19} & $N 1$ & 56 \\
\hline & & & & $N 2$ & 40 \\
\hline & & Pred. & \multirow[t]{2}{*}{5} & $\begin{array}{l}\text { Vocabu } \\
\text { Lary }\end{array}$ & 434 \\
\hline & & Nodes & & $\begin{array}{l}\text { Estt. } \\
\text { Program } \\
\text { Length }\end{array}$ & 82.6 \\
\hline & & \multirow[b]{2}{*}{ Regions } & \multirow[b]{2}{*}{5} & Difficulty & 37.5 \\
\hline & & & & Effort & 16275 \\
\hline & & $\mathrm{V}(\mathrm{G})$ & 6 & Time & 904.16 \\
\hline
\end{tabular}

Consider an example, Let $P$ be the source program in $C$ given below:

Consider a program from fig. 2, the complexity measured by us and computed the complexity of the other proposed measures i.e Lines of Code (LOC), NPATH Complexity (NC), McCabb's complexity (MCC) and Halstead's software science complexity (HSSC) are shown in Table II.

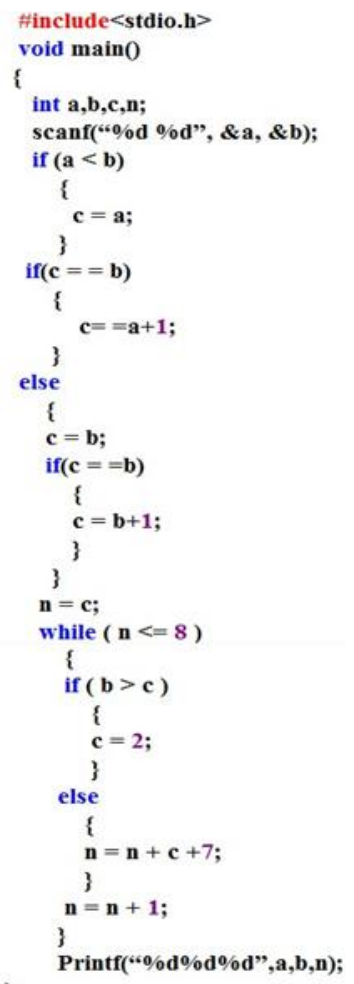

Fig. 2. Source program.

\section{CONCLUSION}

Software complexity metrics have a tendency to be used in judging the quality of software development and one of the vital parts of the SDLC. The volume, control and data based complexity are importance of today's software systems demand the application of effective testing techniques. In addition, it was observed that software complexity metrics which enables the tester to counts the acyclic execution paths 
through a program and improve software quality. This static analysis could be lead to reduce software development cost and improve testing efficacy and software quality by evaluating software complexity metrics with LOC, NPATH (NC), McCabb's complexity metrics (MCC) and Halstead's Software Science Complexity (HSSC).

\section{REFERENCES}

[1] T. J. M. Cabe, "A complexity measure," IEEET Ransactions on Software Engineering, vol. 2, 1976

[2] I. Herraiz, J. M. G. Barahona, and G. Robles, "Towards a theoretical model for software growth," in 29th International Conference on Software Engineering Workshops (ICSEW'07).

[3] W. Harrison, K. Magel, R. Kluczny, and A. Dekok, Applying Software Complexity Metrics to Program Maintenance Compute, vol. 15, pp. 65-79, 1982.

[4] T. D. Marco, "Controlling software projects," Prrntice Hall, New York, 1982.

[5] J. Verner and G. Tate, "A software size model," IEEE Transaction on Software Engineering, vol. 18, no. 4, 1992.

[6] W. Harrision and L. I. Magel, "A complexity based on nesting level," Sigplan Notice, vol. 16, no. 3, 1981.

[7] A. Fitzsimmons and T. Love, "A review and evaluation of software science," Computing Survey, vol. 10, no. 1, March 1978.

[8] S. D. Conte, H. E Dunsmore, and V. Y. Shen, "Software engineering metrics and models," Benjamin/Cummings Publishing Company, Inc., 1986.

[9] B. A. Nejmeh, "NPATH: A measure of execution path complexity and its applications," Comm. of the ACM, vol. 31, no. 2, pp. 188-210, February 1988.

[10] T. A. McCabe, "A complexity measure," IEEE Transactions on Software Engineering, vol. 2, no. 4, pp. 308-320, December 1976.

[11] M. Halstead, Elements of Software Science. North Holland, 1977.

[12] E. E. Millis, "Software metrics," SEI Curriculam Module SEI- CM. vol. 12, no. 2.1, Dec, 1988.

[13] C. M Chung and M. G Yang, "A software maintainability measurement," Proceedings of the 1988 Science, Engineering and Tech. Houston, Texas, pp. V12-16.

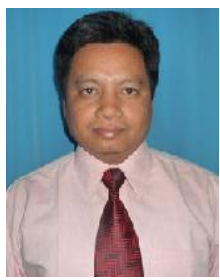

Mrinal Kanti Debbarma was born in Tripura in 1972, Presently working as Assistant Professor at Computer Science and Engineering Department of National Institute of Technology Agartala, India, he received the B.Tech (IET, Lucknow), M.Tech (MNNIT, Allahabad), currently pursuing Ph.D.(Information Technology) at Assam University. His research interest include in Software Engineering with special interest in Software metrics analysis and Regression testing, MANET Routing Protocols, Wireless Sensor
Networks. He has 12 years of academic and 5 years Industrial experience. Mr. Debbarma is a member of IAENG, IACSIT. email: mrinal@ nita.ac.in.

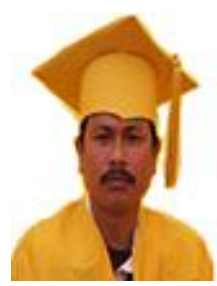

Swapan Debbarma obtained his B.Tech degree in Computer Science and Engineering in 2000 from NERIST (North Eastern Regional Institute of Technology, Deem University, AP, India).In 2010, M.Tech degree in the same field of Engineering from Tripura University and pursuing his $\mathrm{PhD}$ degree from NIT, Agartala in the field of Nanotechnology under the supervision of Professor K. P. Ghatak. He published more than a dozen research papers in International peer-reviewed. He joined as Lecturer in 2002 (Then Tripura Engineering College) and Assistant Professor 2006 in the Department of Computer Science and Engineering NIT.

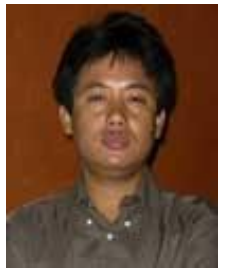

Nikhil Debbarma is an Assistant Professor at the Computer Science and Engineering Department of National Institute of Technology, Agartala, India. He received his B.E. in Computer Science and Engineering at REC Jalandhar, India and M.Tech. in Computer Science and Engineering at National Institute of Technology, Agartala, India. His research interests include OS, Database, Graph Theory, Algorithm.

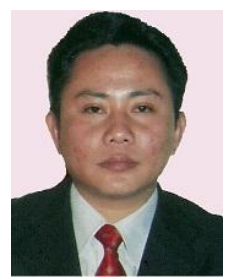

Kunal Chakma is an Assistant Professor at the Computer Science and Engineering Department of National Institute of Technology, Agartala, India. He received his B.E. in Computer Science and Engineering at REC Jalandhar, India and Currently Pursuing M.Tech. in Computer Science and Engineering at National Institute of Technology, Agartala, India. His research interests include Systems Software, Operating Systems, Real-Time Operating Systems, Distributed Systems, Linux.

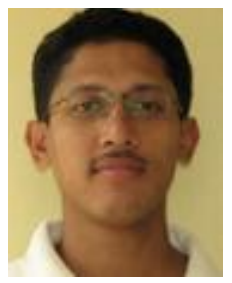

Anupam Jamatia is an Assistant Professor at the Computer Science and Engineering Department of National Institute of Technology, Agartala, India. He received his B.E. in Computer Science and Engineering at University College of Engineering, Burla, Orissa, India in May 2000 and Currently Pursuing M.Tech. in Computer Science and Engineering at National Institute of Technology, Agartala, India. His research interests include Routing Protocol in Wireless Networking and Mobile Computing. He is a member of IEEE. 